\title{
Escarabajos copronecrófagos (coleoptera: scarabaeinae) de la colección entomológica de la universidad de caldas (CEBUC)
}

Título corto: Escarabajos copronecrófagos de la CEBUC

\section{Copronecrophagus beetles (coleoptera: scarabaeinae) from the Entomological Collection of the University of Caldas (CEBUC)}

Short title: Copronecrophagous beetles of CEBUC

\author{
Israel Navarro-Quintero ${ }^{1}$ \\ Stefany Gil-González² \\ Diana Fernanda Álvarez-A ${ }^{3}$ \\ Daniela Jaramillo-Rivera \\ Bedir German Martínez- $Q^{5}$ \\ Sebastián Villada-Bedoya ${ }^{6}$
}

' Biólogo. Universidad de Caldas. Manizales, Colombia

E-mail: israel.dung.beetle@gmail.com

2Programa de Biología, Grupo Entomológico de la Universidad de Caldas (GEUC), Grupo de Ecología de Anfibios y Reptiles (GEDAR), Semillero de Ecotoxicología,

Conservación, Biodiversidad y Educación ambiental (ECOBE), Universidad de Caldas. Manizales Colombia. E-mail: stefany.biologiaucaldas@gmail.com

${ }^{3}$ Bióloga, Grupo de Ecología de Anfibios y Reptiles (GEDAR), Universidad de Caldas. Manizales, Colombia. E-mail: alvarezagudelodianafernanda@gmail.com

+ Bióloga, Grupo Entomológico de la Universidad de Caldas (GEUC), Grupo de investigación en Biodiversidad y Recursos Naturales (Bionat), Universidad de Caldas. Manizales, Colombia. E-mail:dan.jaramillo.795@gmail.com

${ }^{5}$ Biólogo, Universidad de Caldas, Manizales, Colombia. E-mail: sbedirge@gmail.com

${ }^{6}$ Red de Ecoetología, Instituto de Ecología, A.C., Xalapa, 9107o, Veracruz, México.E-mail: escarasebas@gmail.com

Historia del artículo

Fecha de recepción:

O7-07-2021

Fecha de aceptación:

21-10-2021

DOI: https://doi.

org $/ 10.47374 /$

novcol.2021.v16.1966 


\section{Resumen}

Las colecciones biológicas son repositorios de información sobre la biodiversidad y cumplen un rol fundamental para docencia e investigación. Entre las 476 colecciones biológicas de Colombia se encuentra la Colección Entomológica del Programa de Biología de la Universidad de Caldas (CEBUC), la cual posee un acervo importante sobre los escarabajos copronecrófagos (Coleoptera: Scarabaeinae) principalmente del Eje Cafetero. Este grupo de insectos es de gran importancia debido a que están involucrados en diversos procesos ecológicos (e.g., ciclaje de nutrientes). Además, son considerados indicadores biológicos de perturbación antrópica, por lo cual, han sido ampliamente estudiados. El objetivo de este trabajo fue realizar la sistematización de los escarabajos copronecrófagos de la CEBUC y contribuir al conocimiento de la subfamilia en Colombia. Se realizó la revisión, identificación taxonómica y sistematización de los ejemplares, y se generaron mapas de distribución. Se encontraron 3751 ejemplares distribuidos en 18 géneros, donde Dichotomius, Onthophagus y Uroxys fueron los más abundantes, mientras que Pseudocanthon, Sulcophanaeus y Cryptocanthon fueron los géneros menos abundantes. Los agroecosistemas ganaderos, la vegetación secundaria y el bosque subandino húmedo fueron los usos del suelo con mayor cantidad de registros; los agroecosistemas de cultivos permanentes fueron los ecosistemas con menor número de registros. La CEBUC alberga casi la mitad de los géneros de escarabajos copronecrófagos reportados para el país, con registros en diferentes ecosistemas transformados y conservados. Por lo tanto, la CEBUC se proyecta como un referente importante para estudios de conservación, taxonómicos, ecológicos y de planificación ambiental en el país.

Palabras clave: biodiversidad, usos del suelo, distribución, Scarabaeinae, Colombia, Neotrópico.

\section{Abstract}

Biological collections are repositories of information on biodiversity and play a fundamental role in research and teaching. Among the 476 biological collections in Colombia, the Entomological Collection of the Biology Program at the University of Caldas (CEBUC), has an important collection on copro-necrophagous beetles (Coleoptera: Scarabaeinae), mainly from the Andean Coffee Belt. This group of insects represent great importance as they are involved in diverse ecological processes (e.g., nutrient cycling). They are also considered biological indicators of anthropogenic disturbance; therefore, they have been extensively studied. We systematized the copro-necrophagous beetles of the CEBUC and contributed to the knowledge of the subfamily in Colombia. We reviewed, identified, and systematized the specimens to finally present distribution maps. We found 3751 specimens distributed in 18 genera. Dichotomius, Onthophagus, and Uroxys were the most abundant, and Pseudocanthon, Sulcophanaeus, and Cryptocanthon were the least common 
genera. Livestock agro-ecosystems, secondary vegetation, and sub-Andean humid forest were locations with the highest of records, while permanent crop agro-ecosystems were the ones with least registry. The CEBUC includes almost half of the copro-necrophagous beetles genera reported for the country, with records in different transformed and conserved ecosystems. CEBUC is then projected to be an important reference for conservation, taxonomic; ecological, and environmental planning studies.

Key words: biodiversity, soil use, distribution, Scarabaeinae, Colombia, Neotropic.

\section{Introducción}

Las colecciones biológicas constituyen un patrimonio invaluable para el conocimiento de la biodiversidad (Vallejo y Acosta, 2005). Su misión es documentar y conservar ejemplares de diferentes grupos de estudio (e.g., plantas, animales) (Rodríguez et al., 2004; Winker, 2004). Es por esto que son consideradas bancos de información y pueden ser definidas como bibliotecas de especímenes, supeditadas a las condiciones de los ejemplares colectados, la conservación y la fiabilidad de sus datos, adquiriendo un gran valor investigativo, educativo y de conocimiento de la biodiversidad (Vallejo y Acosta, 2005; Vélez et al., 2012; Winker, 2004). Por consiguiente, las colecciones biológicas son consideradas el recurso idóneo para comprender fenómenos a nivel ecológico y ambiental, en los que se destaca la influencia del hombre en la biodiversidad, salud y seguridad epidemiológica, delimitación de áreas para la conservación, distribución de organismos en relación con perturbaciones ambientales, cambios en los patrones biológicos e incluso, respuestas biológicas al cambio climático (Hedrick et al., 2020; Pyke y Ehrlich, 2010).

En los últimos años ha aumentado el número de investigaciones basadas en colecciones biológicas, las cuales se han visto favorecidas por la incorporación de herramientas informáticas y el establecimiento de bases de datos digitales (Pyke y Ehrlich, 2010). Utensilios como GBIF y SIB-Colombia han contribuido a la accesibilidad de los datos, sirviendo de apoyo a distintos grupos biológicos, esto mediado por la sistematización y digitalización de los ejemplares colectados y almacenados en las colecciones biológicas (America, 2016; Arbeláez-Cortés et al., 2017; Mora-Ambriz y FuentesMoreno, 2006; Suarez y Tsutsui, 2004).

La Colección Entomológica del programa de Biología de la Universidad de Caldas (CEBUC), fue fundada en 2002 e incluida formalmente en el Registro Nacional de Colecciones Biológicas (RNC) en 2011, bajo el número de registro 188 (Montaño et al., 2012). Hasta junio de 2019 se encuentra sistematizado el 30\% de los ejemplares en el formato DwC (Dias et al., 2019; IAVH, 2020). Actualmente, CEBUC cuenta con una vasta revisión realizada por Montaño et al. (2012), quienes sistematizaron un total de 5.642 individuos de 111 familias para la colección seca y 28.542 individuos de 90 familias para la colección líquida. 
Entre los órdenes sistematizados se encuentran: Blattodea, Coleoptera, Dermaptera, Diptera, Hemiptera, Hymenoptera, Isoptera, Lepidoptera, Mantodea, Megaloptera, Neuroptera, Odonata, Orthoptera y Phasmatodea.

Uno de los grupos de insectos más diversos y ampliamente distribuidos son los coleópteros (Zhang et al., 2018). Dentro de este grupo, los escarabajos copronecrófagos (Coleoptera: Scarabaeinae) son una de las subfamilias de Scarabaeidae más ampliamente estudiadas, debido a que estos insectos son fundamentales para el funcionamiento de los ecosistemas al cumplir diversos servicios ecosistémicos, como la remoción de estiércol, redistribución de nutrientes en el suelo, control de plagas, dispersión secundaria de semillas y la reducción de emisiones de gases de efecto invernadero (Beynon et al., 2015; Nichols et al., 2008; Slade et al., 2016). Los servicios ecosistémicos brindados por los Scarabaeinae son muy valiosos en términos económicos en sistemas productivos como la ganadería (Lopez-Collado et al., 2017). Además, los escarabajos copronecrófagos son considerados como indicadores biológicos de perturbación, debido a su alta sensibilidad a las perturbaciones antrópicas (França et al., 2020; Gómez et al., 2018; Nichols et al., 2008). Por lo tanto, son excelentes modelos para el desarrollo de programas de monitoreo de la biodiversidad (Morales-Castaño y Medina, 2009).

Pese al potencial bioindicador de los Scarabaeinae, este grupo de insectos presenta una gran incertidumbre taxonómica en géneros comunes y abundantes, como Canthidium, Canthon, Dichotomius, Onthophagus y Uroxys (Cupello, 2018; Pardo-Diaz et al., 2019; Rossini et al., 2018; Vieira et al., 2020). Es por esto que los especímenes depositados en las colecciones biológicas pueden considerarse una excelente herramienta para resolver esta incertidumbre taxonómica. Sin embargo, la alta dispersión de los ejemplares tipo en los museos extranjeros ha dificultado la corroboración de Scarabaeinae en el territorio nacional colombiano (GonzálezAlvarado y Medina, 2015). Otra barrera importante está relacionada con la cobertura de muestreo, ya que se presentan grandes vacíos de información con extensas áreas sin registro en Colombia. Actualmente, es incipiente la cantidad de estudios taxonómicos, genéticos y de distribución, lo cual impide conocer con certeza la identidad y distribución de las especies, sin mencionar que gran parte de los trabajos se quedan en literatura gris (Noriega et al., 2015).

Los escarabajos copronecrófagos son uno de los grupos con mejor representación en el acervo de la colección CEBUC, debido a que la incorporación de especímenes fue continua durante los últimos años gracias al aporte de investigaciones recientes (Blandón-Marín, 2020; Cultid-Medina y Martínez-Quintero, 2019; Jaramillo-Rivera, 2020; NavarroQuintero y Salazar-Salinas, 2019). De este modo, la contribución al conocimiento de los escarabajos copronecrófagos por medio de los especímenes que reposan en la colección CEBUC es de vital interés para investigadores interesados en la biología, ecología y taxonomía de este grupo de insectos. Por 
este motivo, este trabajo tuvo como objetivo llevar a cabo la sistematización de los escarabajos copronecrófagos depositados en la CEBUC, con la finalidad de aportar al conocimiento sobre su distribución, cobertura de muestreo y conservación de este grupo en Colombia.

\section{Materiales y Métodos}

La Colección Entomológica de la Universidad de Caldas (CEBUC) se encuentra ubicada en el Museo de Historia Natural de la Universidad de Caldas, Manizales, Colombia y cuenta con más de 436.0oo ejemplares preservados, tanto en colección en seco como en líquido (IAVH, 2020). Para el desarrollo del presente trabajo, se realizó una búsqueda de todos los Scarabaeinae depositados en CEBUC (conservados en líquido y en seco), donde se revisó el estado de los ejemplares, conservación (deterioro por hongos o psocópteros), elementos de montaje (agujas) y etiquetado (etiquetas dañadas, manchadas, realizadas de manera artesanal o desactualizadas). En el caso de los individuos conservados en líquido (alcohol al 96\%) se reemplazó el alcohol. Posteriormente, se siguieron los estándares actuales propuestos por Montaño et al. (2012) para el etiquetado de los especímenes de la colección seca de la CEBUC. Una vez terminado este procedimiento, se realizaron las acciones pertinentes, como limpieza de los ejemplares y reemplazo de elementos como agujas y etiquetas.
Se llevó a cabo la debida curaduría de cada espécimen, se corroboró la identificación taxonómicay se identificación los ejemplares que carecían de identificación. Esto se realizó por medio de claves y descripciones especializadas de géneros con distribución en Colombia (Cultid-Medina et al., 2012; Tarasov y Dimitrov, 2016; Vaz-de-Mello et $a l ., 2011)$. Debido a la gran incertidumbre taxonómica para el grupo en el país, se optó por determinar todos los especímenes a nivel de género. Para establecer las tribus se utilizaron las propuestas de Tarasov y Dimitrov (2016) y Cultid-Medina et al. (2012). Posteriormente, se realizó el etiquetado de los ejemplares siguiendo lo establecido por Montaño et al. (2012) para la colección CEBUC. Con el fin de mejorar la visualización de los datos, la cantidad de ejemplares se dividió en tres grupos: i) más abundantes, ii) medianamente abundantes y iii) menos abundantes.

\section{Sistematización de la información}

Para la sistematización y reingreso de ejemplares a la colección, se acataron los requisitos actuales de la CEBUC en términos de almacenamiento y etiquetado, adicionalmente se suministró la información de los especímenes en las plantillas Darwin Core del Sistema de Información Sobre Biodiversidad de Colombia (SIB-Colombia, 2021). Dicho formato cuenta con una gran cantidad de campos de interés biológico, ofreciendo información de hábitat, fecha, tipo de preservación, identificación taxonómica, entre otros. Además de lo anterior, se elaboró un mapa de ocurrencia de los individuos depositados en la CEBUC, donde se registran 
las diferentes procedencias de los individuos y los usos del suelo en los que fueron colectados. Para ello se tomó la definición de usos del suelo propuesta por el Sistema de Información Ambiental de Colombia (IDEAM), con sus respectivas capas (IDEAM et al., 2017). Para la división política se tomó la información disponible del Geoportal del Departamento Administrativo Nacional de Estadística (DANE). La construcción del mapa se realizó en el programa QGIS 3.4.12 'Madeira' (Team, 2018).

Los usos del suelo contemplados en el presente estudio son producto de la recopilación de diferentes propuestas sobre ecosistemas y biomas en el país (e.g., Chávez y Arango 1998; HernándezCamacho et al. 1992). Además, se incluyó una sólida actualización con diferentes parámetros como geología, geomorfología, clima, coberturas del suelo y biomas. Estos usos del suelo tienen un especial énfasis en la frontera entre coberturas naturales (i.e., bosques) y coberturas transformadas como: agroecosistemas, urbanizaciones, plantaciones forestales, entre otros.

\section{Resultados}

$\mathrm{Al}$ interior de la CEBUC se encontraron 3.751 ejemplares provenientes de tanto de la colección líquida como de la colección seca, distribuidos en ocho tribus (Tarasovy Dimitrov, 2016) y 18 géneros de Scarabaeinae (Anexo 1), provenientes de 13 departamentos de Colombia. Los géneros más abundantes fueron: Dichotomius, Onthophagus y Uroxys (provenientes de nueve, ocho y un departamento, respectivamente). Los géneros medianamente abundantes fueron: Canthidium, Sylvicanthon y Deltochilum (provenientes de cinco, tres y seis departamentos, respectivamente). Por último, los géneros menos abundantes fueron: Pseudocanthon, Sulcophanaeus y Cryptocanthon (todos del departamento de Caldas) (Tabla 1).

Los individuos depositados en CEBUC estuvieron distribuidos en trece departamentos de Colombia, así: Caldas, 17 géneros; Valle del Cauca, nueve; Chocó y Antioquia, ocho; Meta, siete; Vaupés, seis; Quindío y Risaralda, cuatro; Cauca, Tolima y Nariño, dos; y Bolívar y Putumayo, un género (Figura 1).

En cuanto a los usos del suelo, el agroecosistema ganadero (Ag) fue el que contó con la mayor cantidad de ejemplares (n) y géneros $(\mathrm{g})(\mathrm{n}=2086 ; \mathrm{g}=17$, respectivamente), seguido por vegetación secundaria (Vs) (n $=855 ; g=13)$ y bosque subandino húmedo (Bsh) $(\mathrm{n}=188 ; \mathrm{g}=10)$. Los usos del suelo con cantidades medias de ejemplares y géneros fueron: bosque basal húmedo (Bbh) $(\mathrm{n}=105 ; \mathrm{g}=9$, respectivamente), el agroecosistema de mosaico de cultivos, pastos y espacios naturales (Ae) $(\mathrm{n}=97 ; \mathrm{g}=11)$ y el agroecosistema forestal $(\mathrm{Af})(\mathrm{n}=83 ; \mathrm{g}=4)$. Por último, los ecosistemas que contaron con la menor cantidad de ejemplares y géneros fueron: río de aguas blancas (Rab), bosque andino húmedo (Bah) y agroecosistema de cultivos permanentes (Ap), todos con un solo ejemplar de los géneros Onthophagus, Dichotomius y Oxysternon respectivamente (Tabla 2; Figura. 1). 
Tabla 1. Géneros de escarabajos copronecrófagos depositados en CEBUC, colectados en diferentes departamentos de Colombia.

\begin{tabular}{|c|c|c|c|c|c|c|c|c|c|c|c|c|c|c|c|c|}
\hline \multirow[b]{2}{*}{ Tribu } & \multirow[b]{2}{*}{ Género } & \multirow[b]{2}{*}{$\tilde{d}$} & \multicolumn{13}{|c|}{ Departamento } & \multirow[b]{2}{*}{ 章 } \\
\hline & & & 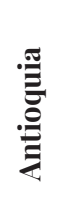 & 竞 & $\frac{\mathfrak{E}}{\tilde{J}}$ & تِّ & 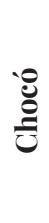 & $\frac{\pi}{\frac{\pi}{2}}$ & 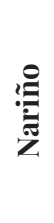 & 玄 & 。․ㅡ & 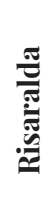 & 兽 & 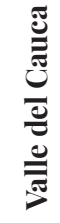 & 兽 & \\
\hline Ateuchini & Scatimus & A & o & $\mathrm{O}$ & 39 & o & $\mathrm{O}$ & $\mathrm{O}$ & o & o & $\mathrm{O}$ & $\mathrm{O}$ & $\mathrm{O}$ & $\mathrm{O}$ & $\mathrm{O}$ & 39 \\
\hline \multirow[t]{3}{*}{ Coprini } & Canthidium & B & $\mathrm{O}$ & $\mathrm{O}$ & 274 & o & 1 & $\mathrm{O}$ & $\mathrm{O}$ & $\mathrm{O}$ & 1 & $\mathrm{O}$ & $\mathrm{O}$ & 13 & 20 & 309 \\
\hline & Ontherus & C & $\mathrm{O}$ & $\mathrm{O}$ & 297 & 1 & $\mathrm{O}$ & 3 & 2 & o & 1 & 2 & $\mathrm{O}$ & $\mathrm{O}$ & 8 & 314 \\
\hline & Uroxys & $\mathrm{D}$ & $\mathrm{O}$ & O & 502 & $\mathrm{O}$ & $\mathrm{O}$ & $\mathrm{O}$ & $\mathrm{O}$ & o & $\mathrm{O}$ & $\mathrm{O}$ & $\mathrm{O}$ & $\mathrm{O}$ & $\mathrm{O}$ & 502 \\
\hline \multirow[t]{5}{*}{ Deltochilini } & Canthon & $\mathrm{E}$ & 2 & $\mathrm{O}$ & 288 & o & $\mathrm{O}$ & 3 & o & 1 & $\mathrm{O}$ & $\mathrm{O}$ & o & 12 & 67 & 373 \\
\hline & Deltochilum & $\mathrm{F}$ & 1 & O & 115 & o & 2 & 4 & o & o & o & 1 & O & 47 & o & 170 \\
\hline & Pseudocanthon & G & o & $\mathrm{O}$ & 2 & o & $\mathrm{O}$ & $\mathrm{O}$ & o & o & o & $\mathrm{O}$ & $\mathrm{O}$ & O & o & 2 \\
\hline & Scybalocanthon & $\mathrm{H}$ & $\mathrm{O}$ & $\mathrm{O}$ & $\mathrm{O}$ & $\mathrm{O}$ & o & $\mathrm{O}$ & 1 & o & $\mathrm{O}$ & $\mathrm{O}$ & $\mathrm{O}$ & $\mathrm{O}$ & 2 & 3 \\
\hline & Sylvicanthon & I & 3 & $\mathrm{O}$ & 235 & o & 6 & $\mathrm{O}$ & $\mathrm{O}$ & o & o & $\mathrm{O}$ & $\mathrm{O}$ & $\mathrm{O}$ & $\mathrm{O}$ & 244 \\
\hline Dermariziellini & Cryptocanthon & $\mathrm{J}$ & $\mathrm{O}$ & $\mathrm{O}$ & 1 & $\mathrm{O}$ & $\mathrm{O}$ & $\mathrm{O}$ & $\mathrm{O}$ & $\mathrm{O}$ & $\mathrm{O}$ & $\mathrm{O}$ & $\mathrm{O}$ & $\mathrm{O}$ & $\mathrm{O}$ & 1 \\
\hline Dichotomiini & Dichotomius & K & 5 & 1 & 876 & o & 1 & 36 & $\mathrm{O}$ & $\mathrm{O}$ & 1 & 2 & $\mathrm{O}$ & 5 & 1 & 928 \\
\hline Eurysternini & Eurysternus & $\mathrm{L}$ & o & $\mathrm{O}$ & 69 & $\mathrm{O}$ & 1 & 18 & o & o & $\mathrm{O}$ & $\mathrm{O}$ & $\mathrm{O}$ & 4 & $\mathrm{O}$ & 92 \\
\hline \multirow[t]{2}{*}{ Onthophagini } & Digitonthophagus & M & $\mathrm{O}$ & $\mathrm{O}$ & 7 & o & o & o & o & o & $\mathrm{O}$ & $\mathrm{O}$ & $\mathrm{O}$ & o & $\mathrm{O}$ & 7 \\
\hline & Onthophagus & $\mathrm{N}$ & 2 & O & 604 & o & 2 & 58 & o & o & o & $\mathrm{O}$ & 1 & 8 & 4 & 679 \\
\hline \multirow[t]{5}{*}{ Phanaeini } & Coprophanaeus & $\mathrm{O}$ & 1 & o & 30 & o & 3 & $\mathrm{O}$ & o & o & o & $\mathrm{O}$ & $\mathrm{O}$ & 4 & o & 38 \\
\hline & Oxysternon & $\mathrm{P}$ & 1 & $\mathrm{O}$ & 20 & 1 & 1 & $\mathrm{O}$ & $\mathrm{O}$ & o & 1 & 1 & 1 & 2 & o & 28 \\
\hline & Phanaeus & $Q$ & 1 & O & 6 & $\mathrm{O}$ & $\mathrm{O}$ & 2 & $\mathrm{O}$ & o & o & $\mathrm{O}$ & $\mathrm{O}$ & 11 & o & 20 \\
\hline & Sulcophanaeus & $\mathrm{R}$ & o & $\mathrm{O}$ & 2 & o & o & $\mathrm{O}$ & o & o & $\mathrm{O}$ & $\mathrm{O}$ & $\mathrm{O}$ & $\mathrm{O}$ & $\mathrm{O}$ & 2 \\
\hline & Total & & 16 & 1 & 3367 & 2 & 17 & 124 & 3 & 1 & 4 & 6 & 2 & 106 & 102 & 3751 \\
\hline
\end{tabular}




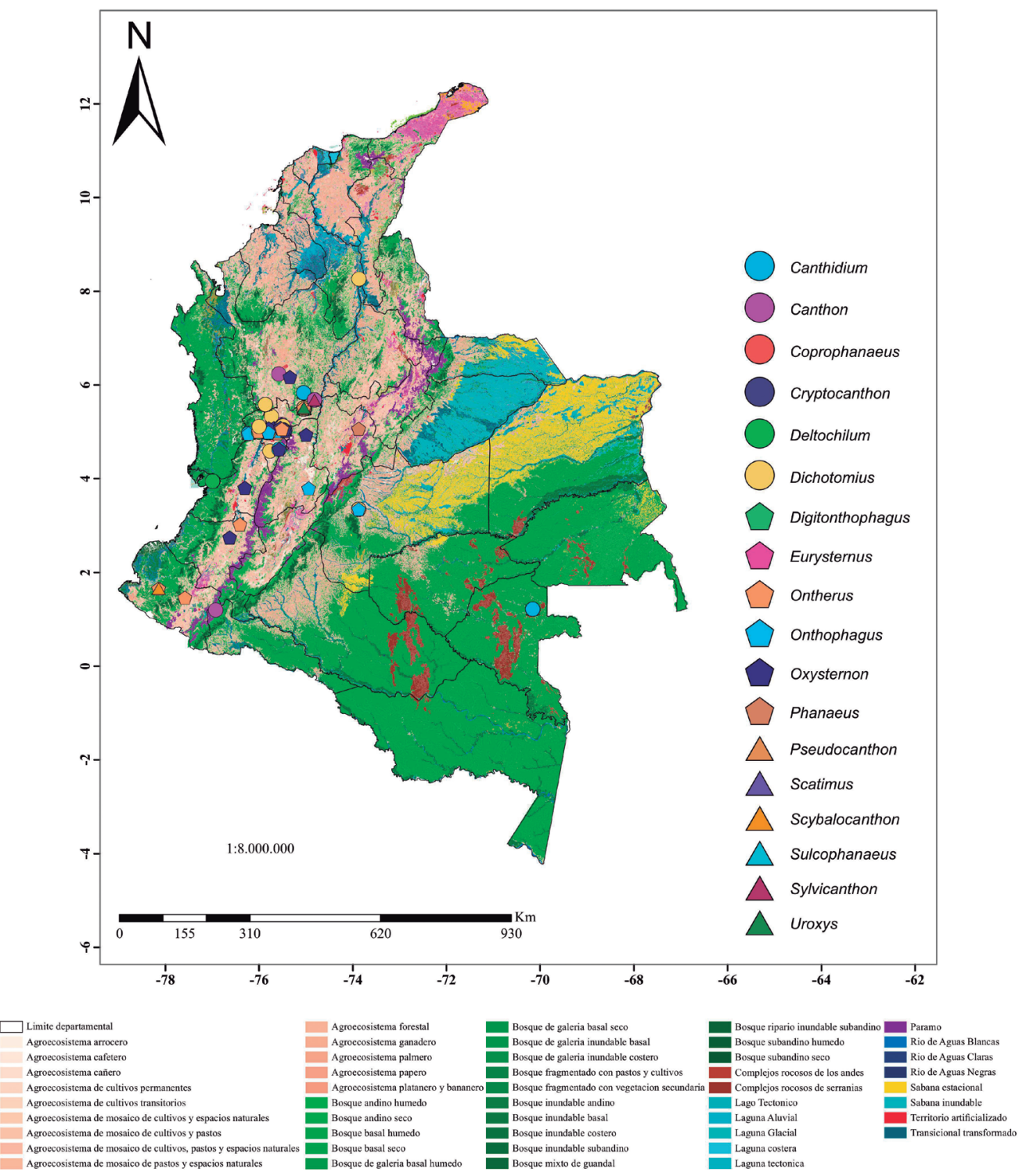

Fig. 1. Distribución en Colombia de los Scarabaeinae depositados en la Colección Entomológica de la Universidad de Caldas (CEBUC). Se presentan los usos del suelo donde fueron colectados los especímenes. 
Tabla 2. Géneros de escarabajos copronecrófagos depositados en CEBUC y colectados en diferentes usos del suelo de Colombia. $\mathrm{Ac}=$ agroecosistema cafetero, $\mathrm{Ae}=$ agroecosistema

de mosaico de cultivos, pastos y espacios naturales, $\mathrm{Af}=$ agroecosistema forestal, $\mathrm{Ag}=$ agroecosistema ganadero, $\mathrm{Al}=$ agroecosistema palmero, $\mathrm{Am}=$ agroecosistema de mosaico de cultivos y pastos, $\mathrm{Ap}=$ agroecosistema de cultivos permanentes, Bbh = bosque basal húmedo, Bha $=$ bosque andino húmedo, Bsh = bosque subandino húmedo, Rab = río de aguas blancas, $\mathrm{Ta}=$ territorio artificializado, $\mathrm{Tt}=$ transicional transformado $\mathrm{y} \mathrm{Vs}=$ vegetación secundaria .

\begin{tabular}{|c|c|c|c|c|c|c|c|c|c|c|c|c|c|c|c|c|c|}
\hline \multirow{2}{*}{ Tribu } & \multirow{2}{*}{ Género } & \multirow{2}{*}{ ש̇ } & \multicolumn{14}{|c|}{ Ecosistema } & \multirow{2}{*}{ 흘 } \\
\hline & & & Ac & Ae & Af & Ag & Al & Am & Ap & Bbh & Bha & Bsh & Rab & Ta & $\mathbf{T t}$ & Vs & \\
\hline Ateuchini & Scatimus & A & o & $\mathrm{O}$ & $\mathrm{O}$ & 38 & $\mathrm{O}$ & o & O & $\mathrm{O}$ & o & $\mathrm{O}$ & $\mathrm{O}$ & o & $\mathrm{O}$ & 1 & 39 \\
\hline \multirow[t]{3}{*}{ Coprini } & Canthidium & B & $\mathrm{O}$ & o & 3 & 144 & o & 1 & o & 13 & $\mathrm{O}$ & 5 & $\mathrm{O}$ & 5 & $\mathrm{O}$ & 138 & 309 \\
\hline & Ontherus & $\mathrm{C}$ & 1 & 38 & 27 & 90 & 3 & 3 & $\mathrm{O}$ & $\mathrm{O}$ & o & $\mathrm{O}$ & $\mathrm{O}$ & 42 & 1 & 109 & 314 \\
\hline & Uroxys & $\mathrm{D}$ & $\mathrm{O}$ & $\mathrm{O}$ & 23 & 202 & $\mathrm{O}$ & $\mathrm{O}$ & $\mathrm{O}$ & $\mathrm{O}$ & $\mathrm{O}$ & 41 & $\mathrm{O}$ & 3 & $\mathrm{O}$ & 233 & 502 \\
\hline \multirow[t]{5}{*}{ Deltochilini } & Canthon & $\mathrm{E}$ & $\mathrm{O}$ & 1 & $\mathrm{O}$ & 262 & 3 & o & o & 12 & o & 10 & o & 7 & o & 78 & 373 \\
\hline & Deltochilum & $\mathrm{F}$ & $\mathrm{O}$ & 4 & $\mathrm{O}$ & 40 & 4 & o & $\mathrm{O}$ & 47 & o & 21 & $\mathrm{O}$ & 17 & o & 37 & 170 \\
\hline & Pseudocanthon & $\mathrm{G}$ & O & o & 0 & 2 & o & O & 0 & o & O & o & O & O & o & O & 2 \\
\hline & Scybalocanthon & $\mathrm{H}$ & o & o & o & 2 & o & o & o & o & o & o & $\mathrm{O}$ & o & 1 & o & 3 \\
\hline & Sylvicanthon & I & o & 3 & $\mathrm{O}$ & 229 & o & $\mathrm{O}$ & o & $\mathrm{O}$ & $\mathrm{O}$ & 3 & $\mathrm{O}$ & 3 & $\mathrm{O}$ & 6 & 244 \\
\hline Dermariziellini & Cryptocanthon & $\mathrm{J}$ & $\mathrm{O}$ & $\mathrm{O}$ & $\mathrm{O}$ & 1 & $\mathrm{O}$ & o & o & o & o & o & o & o & o & o & 1 \\
\hline Dichotomiini & Dichotomius & K & 1 & 12 & $\mathrm{O}$ & 625 & 36 & 3 & $\mathrm{O}$ & 5 & 1 & 29 & $\mathrm{O}$ & 44 & $\mathrm{O}$ & 172 & 928 \\
\hline Eurysternini & Eurysternus & $\mathrm{L}$ & o & 1 & $\mathrm{O}$ & 39 & 18 & $\mathrm{O}$ & $\mathrm{O}$ & 4 & $\mathrm{O}$ & 7 & $\mathrm{O}$ & 4 & o & 19 & 92 \\
\hline \multirow[t]{2}{*}{ Onthophagini } & Digitonthophagus & M & $\mathrm{O}$ & $\mathrm{O}$ & $\mathrm{o}$ & 6 & $\mathrm{o}$ & $\mathrm{O}$ & $\mathrm{O}$ & $\mathrm{O}$ & $\mathrm{O}$ & 1 & $\mathrm{O}$ & $\mathrm{O}$ & $\mathrm{O}$ & $\mathrm{O}$ & 7 \\
\hline & Onthophagus & $\mathrm{N}$ & 2 & 27 & 30 & 377 & 58 & o & $\mathrm{O}$ & 8 & $\mathrm{O}$ & 70 & 1 & 54 & o & 52 & 679 \\
\hline \multirow[t]{5}{*}{ Phanaeini } & Coprophanaeus & $\mathrm{O}$ & o & 1 & $\mathrm{O}$ & 23 & $\mathrm{O}$ & 1 & $\mathrm{O}$ & 4 & $\mathrm{O}$ & $\mathrm{O}$ & o & 4 & $\mathrm{O}$ & 5 & 38 \\
\hline & Oxysternon & $\mathrm{P}$ & 4 & 7 & $\mathrm{O}$ & 2 & o & 5 & 1 & 1 & $\mathrm{O}$ & 1 & $\mathrm{O}$ & 3 & $\mathrm{O}$ & 4 & 28 \\
\hline & Phanaeus & $\underline{Q}$ & 1 & 2 & $\mathrm{O}$ & 4 & 1 & $\mathrm{O}$ & $\mathrm{O}$ & 11 & o & $\mathrm{O}$ & $\mathrm{O}$ & o & $\mathrm{O}$ & 1 & 20 \\
\hline & Sulcophanaeus & $\mathrm{R}$ & o & 1 & $\mathrm{O}$ & $\mathrm{O}$ & $\mathrm{O}$ & $\mathrm{O}$ & $\mathrm{O}$ & o & $\mathrm{O}$ & $\mathrm{O}$ & $\mathrm{O}$ & 1 & $\mathrm{O}$ & o & 2 \\
\hline & Total & & 9 & 97 & 83 & 2086 & 123 & 13 & $\mathbf{1}$ & 105 & $\mathbf{1}$ & 188 & $\mathbf{1}$ & 187 & 2 & 855 & 3751 \\
\hline
\end{tabular}


Los géneros más abundantes estuvieron presentes en una gran cantidad de usos del suelo. Dichotomius y Onthophagus fueron registrados en diez usos del suelo, pero predominaron en agroecosistema ganadero (Ag), mientras que Uroxys estuvo en cinco ecosistemas, pero fue dominante en vegetación secundaria (Vs). En cuanto a los géneros medianamente abundantes, Canthidium y Sylvicanthon fueron registrados en siete ecosistemas, mientras que Deltochilum fue registrado en cinco; al igual que Dichotomius y Onthophagus, estos tres géneros fueron dominantes en agroecosistema ganadero (Ag). En el caso de Deltochilum, estuvo asociado principalmente al bosque basal húmedo (Bbh). Finalmente, los géneros menos abundantes tuvieron registros en muy pocos ecosistemas, por ejemplo, Pseudocanthon y Cryptocanthon fueron registrados en agroecosistema ganadero (Ag), mientras que Sulcophanaeus solo se registró en territorio artificial (Ta) y agroecosistema de mosaico de cultivos, pastos y espacios naturales (Ae) (Figura 2).

En la CEBUC reposan especímenes que datan desde el año 1993, con dos ejemplares de este año, una década después se deposita un ejemplar más y continúa así por los dos años siguientes. No fue sino hasta 2006 que se presentó el ingreso de 6o ejemplares (Figura 3). A partir de ese

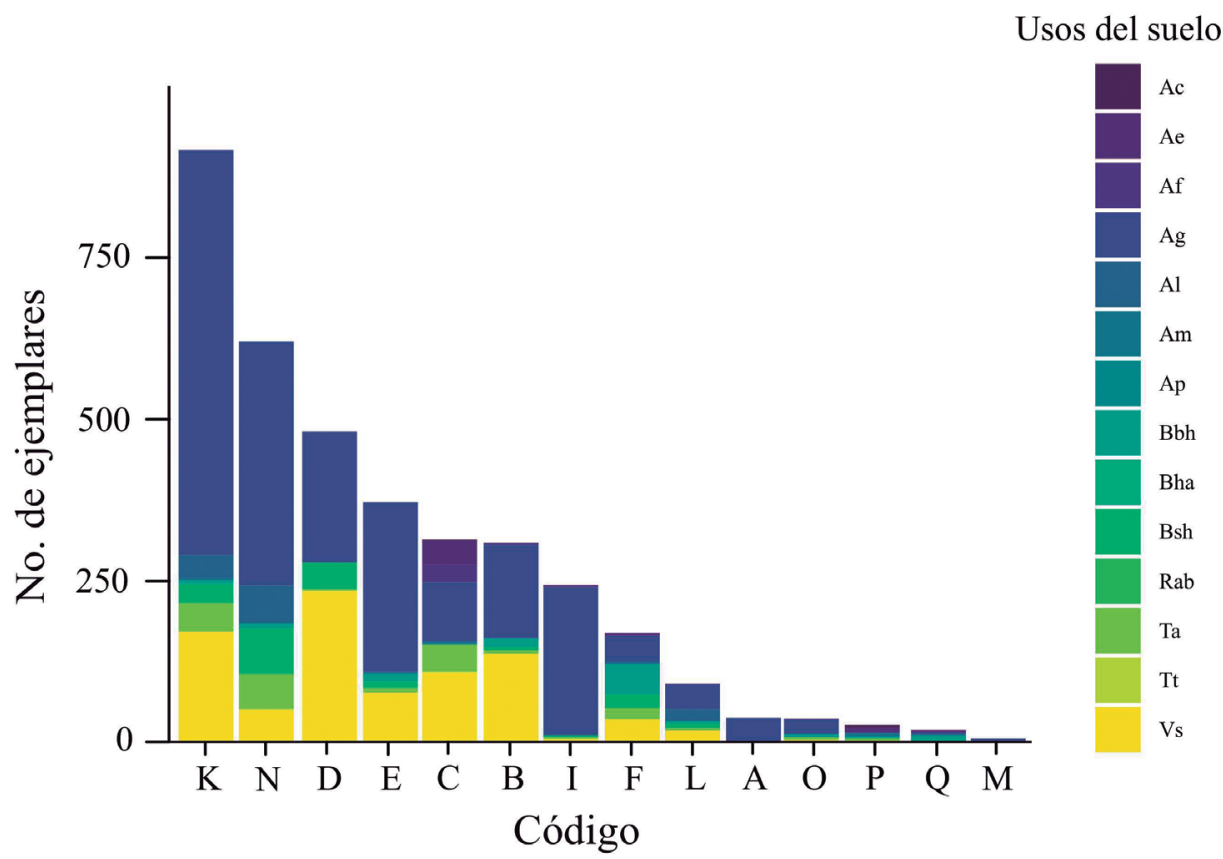

Figura 2. Géneros de escarabajos copronecrófagos depositados en CEBUC y colectados en diferentes ecosistemas de Colombia (ver los códigos de los géneros en las Tablas 1 y 2). 
año, el número de especímenes aumentó exponencialmente debido a los diferentes proyectos de investigación, trabajos de grado y salidas académicas realizados por investigadores y estudiantes interesados en los Scarabaeinae. Cabe resaltar que la mayor cantidad de ejemplares que alberga la CEBUC proviene del departamento de Caldas, sin embargo, también cuenta con una buena representación de otros departamentos como Meta, Valle del Cauca y Vaupés (Figura 3).

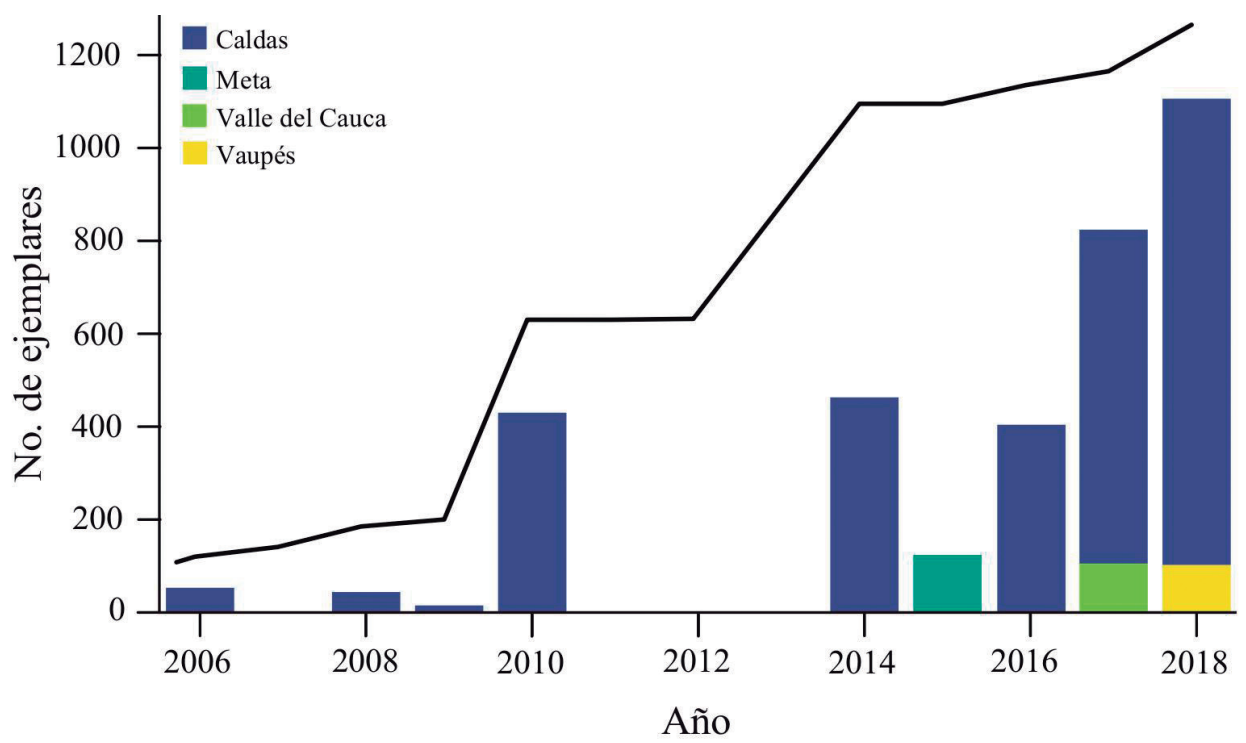

Figura 3: Escala temporal del depósito de ejemplares en CEBUC indicando los departamentos con mayor número de registros de escarabajos copronecrófagos (ver Tabla 1).

\section{Discusión}

El número de ejemplares de escarabajos copronecrófagos depositados en la colección CEBUC corresponde al 45\% (18 géneros) de los géneros reportados para Colombia (40 en total) (Noriega et al., 2015). La falta de revisiones taxonómicas para algunos géneros de Scarabaeinae distribuidos en Colombia de grupos altamente representados en las colecciones dificulta la determinación precisa de los ejemplares
(González-Alvarado y Medina, 2015). Según González-Alvarado y Medina (2015), aproximadamente el 51\% de las especies de escarabajos copronecrófagos en Colombia no tienen identificación a nivel de especie, debido a esta limitante, en el presente trabajo se decidió presentar los resultados de los escarabajos copronecrófagos hasta el nivel taxonómico de género y de este modo no incurrir en identificaciones imprecisas a nivel de morfoespecie. 
El género Dichotomius presentó el mayor número de ejemplares al interior de la colección CEBUG, esto puede deberse a que es uno de los géneros de Scarabaeinae con mayor riqueza de especies en el hemisferio occidental y en Suramérica (Nunes y Vazde-Mello, 2019). Además, presentan altas abundancias en hábitats contrastantes como pasturas y bosques, y es un género con muchas especies de hábitos generalistas en términos de uso de hábitat, debido a que pueden estar en diferentes coberturas como bosques, pastizales, matorrales y cultivo (Cultid-Medina et al., 2012). Esto concuerda con los resultados de los usos del suelo, donde el agroecosistema ganadero (Ag) fue el que presentó la mayor cantidad de ejemplares, siendo Dichotomius el género más abundante. Por otra parte, los géneros Pseudocanthon, Sulcophanaeus y Cryptocanthon se encuentran pobremente representados en la colección, lo que podría deberse a que el acervo de la CEBUC cuenta principalmente con ejemplares provenientes de sistemas antropizados y estos géneros normalmente se encuentran asociados a zonas bien conservadas (Braga et al., 2013). Aunque los géneros Pseudocanthon y Sulcophanaeus están ampliamente distribuidos en Colombia en bosques en buen estado de conservación (Cultid-Medina et al., 2012), en CEBUC reposan pocos individuos, posiblemente a raíz del bajo esfuerzo de muestreo en las zonas de distribución de estos géneros. La baja representación de Cryptocanthon en la CEBUC, posiblemente se deba a que las especies de este género son raras en las colectas y los especímenes colectados en Colombia son de distribución Andina; de este género se conocen 15 especies, aunque su ecología ha sido poco estudiada (Arias y Medina, 2014; Martínez-Revelo et al., 2020).

Para la región del Eje Cafetero existe una alta riqueza de Scarabaeinae al contar con 19 de los 40 géneros reportados para el país (Cultid-Medina et al., 2014). En CEBUC hay registros de 17 de los 19 géneros reportados para esta región y específicamente para el departamento de Caldas se reportan 16 géneros. Esto concuerda con Noriega et al. (2015), quienes reportan que las zonas mejor muestreadas de Colombia en comparación con otras regiones se encuentran en la región Andina. No obstante, el departamento de Caldas presenta un nivel de muestreo intermedio-bajo, con pocas localidades y con extensas áreas sin ningún registro; sumado a esto, el alto grado de incertidumbre taxonómica interfiere en gran medida a la hora de llevar a cabo estudios comparativos usando a los Scarabaeinae como modelo a través de diferentes escalas espaciales (Cultid-Medina et al., 2014; Noriega et al., 2015). Por lo tanto, la información almacenada en la colección CEBUC es de vital interés, dado que hay una gran representatividad de los escarabajos copronecrófagos del departamento de Caldas. Además, varios registros provienen de localidades nuevas y de difícil acceso, lo que constituye información regional valiosa que puede ser usada en la planificación de estudios de conservación en el departamento de Caldas. De acuerdo con los ecosistemas propuestos por IDEAM et al. (2007), la mayoría de géneros provienen de Ag, Bbh, Bha y Bsh, que en conjunto aportan 17 géneros, esto concuerda con los esfuerzos de diferentes instituciones del país por estudiar bosques 
húmedos de tierras bajas, premontanos y montanos (Noriega et al., 2015).

El incremento en el ingreso de especímenes a la CEBUC, específicamente en los años 2010, 2014 y 2018, coincide con un análisis histórico realizado por Noriega et al. (2015), donde encontraron un importante crecimiento en el número de trabajos de investigación en más del 200\% en el periodo 2000-2005 y en más del 200\% en el periodo 2006-2014. Los ejemplares depositados en CEBUC provienen de una amplia variedad de usos del suelo, pero la gran mayoría provienen de dos localidades: i) Reserva Natural de Río Manso, ubicada al Oriente de Caldas, que ha aportado una alta cantidad de Scarabaeinae a la colección al estar relacionada con numerosas salidas de campo realizadas por el programa de Biología de la Universidad de Caldas; ii) Parque Nacional Natural Selva de Florencia (Samaná), donde se colectó un gran número de ejemplares como resultado del componente entomológico del proyecto "Caldas Expedición Siglo XXI (CESXXI)". Cabe resaltar que este lugar es de las pocas áreas que cuentan con bosques bien conservados en el departamento (Ballesteros et al., 2009). La buena representación de escarabajos copronecrófagos del PNN Selva de Florencia depositados en la CEBUC contribuye y fortalece el conocimiento de esta zona conservada y poco explorada del país, aportando una base para trabajos enmarcados en taxonomía, ecología y conservación.

Este trabajo resalta la importancia de conservar y mantener las colecciones biológicas, lo cual asegura que el conocimiento perdure por generaciones, de manera que se siga transmitiendo y comunicando (Vélez et al., 2012). Asimismo, fortalece el conocimiento acerca de los escarabajos copronecrófagos de la región, lo que posibilita conocer más acerca de su distribución, ecología, rasgos de historia de vida, dinámicas poblacionales, comportamiento, e incluso, saber hacia dónde dirigir herramientas de planificación para esfuerzos de conservación, que a su vez representa una gran oportunidad para el desarrollo de futuras investigaciones. La importancia que tiene GEBUC en la región como un repositorio de información, será de gran utilidad para futuros estudios, debido a que cuenta con una cantidad importante de ejemplares provenientes de ecosistemas ganaderos, esenciales a la hora de realizar estudios de impacto y planeación de agroecosistemas.

\section{Conclusiones}

En Colombia existe un alto potencial para el estudio de Scarabaeinae en zonas inexploradas y de difícil acceso. Por lo tanto, evaluar y documentar la diversidad biológica presente en estas áreas poco estudiadas, es un tema de vital interés para el conocimiento de la biodiversidad de Scarabaeinae en Colombia. Mucha de la información de los inventarios de diversidad biológica reposa en colecciones biológicas, por esto es primordial que dicha información este bien sistematizada y actualizada. De este modo, la sistematización de los escarabajos copronecrófagos de la CEBUC contribuye al conocimiento del grupo en la región y en Colombia, pues alberga una gran cantidad de ejemplares y géneros de Scarabaeinae 
provenientes de una amplia variedad de usos del suelo, que abarcan áreas transformadas y conservadas. Sin embargo, la identidad de las especies aún sigue siendo un gran obstáculo para el estudio de estos organismos, lo cual repercute a la hora de realizar estudios ecológicos, biogeográficos y de conservación para el grupo. El esfuerzo concentrado de muestreo en algunas regiones (e.g., área Andina) y ecosistemas (e.g.., bosques húmedos y agroecosistemas), lleva a que se sigan presentando sesgos. Todo lo anterior, más que una limitante, debe verse como una puerta abierta para futuras investigaciones que aborden temas problemáticos, como el de la incertidumbre taxonómica de muchos géneros del grupo con distribución Neotropical.

\section{Agradecimientos}

Los autores agradecemos al proyecto "Evaluación de la estructura genética poblacional y conectividad funcional de un paisaje cafetero para dos especies de escarabajos coprófagos (Coleoptera: Scarabaeinae): Aplicación de la genética del paisaje para entender el papel de los escenarios dominados por café de sol en el manejo y la conservación de la biodiversidad en los Andes occidentales de Colombia” (código: 1127-714-51264) financiado por Colciencias. Al proyecto "Caldas Expedición Siglo XXI Fase I: Levantamiento de la biota y geología de una subcuenca del Parque Nacional Natural Selva de Florencia" (Código: 1421717). Al Laboratorio de Colecciones Biológicas de la Universidad de Caldas, por prestar el espacio para el desarrollo de este trabajo. A la profesora Lucimar Gomes Dias por sus aportes y comentarios al manuscrito. Al grupo de investigación en Biodiversidad y Recursos Naturales (BIONAT), y a Luis Fernando Salazar Salinas por su apoyo en el procesamiento de muestras y comentarios.

\section{Referencias}

America, E. S. A. 2016. ESA Position Statement on the Importance of Entomological Collections. Ann. Entomol. Soc. Am, 110: 565-566. 
Arbeláez-Cortés, E., Acosta-Galvis, A. R., DoNascimiento, C., Espitia-Reina, D., González-Alvarado, A., Medina, C. A. 2017. Knowledge linked to museum specimen vouchers: measuring scientific production from a major biological collection in Colombia. Scientometrics, 112(3), 1323-1341. Disponible en: http://dx.doi.org/10.1007/S11192-017-2461-4

Arias, J. A., Medina, C. A. 2014. Tres nuevas especies de Cryptocanthon Balthasar, 1942 (Coleoptera: Scarabaeidae: Scarabaeinae) para Colombia. Caldasia, 36(1), 165-180. Disponible en: https://doi.org/10.15446/caldasia.v36n1.43898

Ballesteros, H., Arroyave, J., Walker, R., Echeverry, L., Acosta, H., Betancourt, A., Diaz-Mesa, J., Lopez, M., Moreno-Ortiz, E., Villegas, H. 2009. Plan de manejo 2008-2012 Parque Nacional Natural Selva de Florencia. Corregimiento de FlorenciaSamaná (Caldas): Parques Nacionales Naturales de Colombia.

Beynon, S. A., Wainwright, W. A., Christie, M. 2015. The application of an ecosystem services framework to estimate the economic value of dung beetles to the UK cattle industry. Ecological Entomology, 40, 124-135. Disponible en: https://doi. org/10.1111/een.12240

Blandón-Marín, G. 2020. Estructura genómica poblacional de Ontherus lunicollis (Coleoptera: Scarabaeinae) en un paisaje cafetero fragmentado en los Andes colombianos. Trabajo de Grado en Maestría en Ciencias Biológicas, Universidad de Caldas, Facultad de Ciencias Exactas y Naturales, Posgrados, Manizales - Caldas.

Chávez, M., Arango, N. 1998. Informe Nacional sobre el Estado de la Biodiversidad-Colombia. Tomo I. Causas de la pérdida de biodiversidad. Instituto de Investigación de Recursos Biológicos Alexander von Humboldt, PNUMA, Ministerio del Medio Ambiente. Bogotá, Colombia. 
Cultid-Medina, C., Lobo, J. M., Medina, C. A., González, F. A., Escobar, F., Chacón de Ulloa, P. 2014. Completitud del inventario de escarabajos coprófagos (Coleoptera: Scarabaeinae) en la Ecorregión del Eje Cafetero, Colombia. Revista Colombiana de entomología, 40(1):111-119.

Cultid-Medina, C., Martínez-Quintero, B. 2019. More than 5years! an unusually long-lived dung beetle (Scarabaeinae) in an Andean agricultural landscape. Neotropical entomology, 48(3), 522-526. Disponible en: https://doi.org/10.1007/s13744-019-00673-w

Cultid-Medina, C., Medina, C., Martínez, B., Escobar, A., Constantino, L., Betancur, N. 2012. Escarabajos coprófagos (Scarabaeinae) del Eje Cafetero: guía para el estudio ecológico. WCS-Colombia, CENICAFÉ y Federación Nacional de Cafeteros. Villa María. Colombia.

Cupello, M. 2018. On the types species of the New World dung beetle genus Canthidium Erichson, 1847 (Coleoptera: Scarabaeidae: Scarabaeinae), with an annotated checklist of species. Zootaxa, 4388(4), 451-486. Disponible en: https://doi. org/10.11646/zootaxa.4388.4.1

Dias, L. G., Henao, E. R., Campaz, M. M. 2019. Colección Entomológica del Programa de Biología-Universidad de Caldas (CEBUC).

França, F. M., Ferreira, J., Vaz-de-Mello, F. Z., Maia, L. F., Berenguer, E., Ferraz Palmeira, A., Fadini, R., Louzada, J., Braga, R., Hugo Oliveira, V. 2020. El Niño impacts on humanmodified tropical forests: Consequences for dung beetle diversity and associated ecological processes. Biotropica, 52(2), 252-262. Disponible en: https://doi.org/10.1111/btp.12756

Gómez, V. G., Verdú, J. R., Alonso, G. G., Zurita, G. A. 2018. Relationship between land uses and diversity of dung beetles (Coleoptera: Scarabaeinae) in the southern Atlantic forest of Argentina: which are the key factors? Biodiversity and Conservation, 27(12), 3201-3213. Disponible en: https://oi. org/10.1007/S10531-018-1597-8 
González-Alvarado, A., Medina, C. A. 2015. Listado de especies de escarabajos coprófagos (Coleoptera: Scarabaeidae: Scarabaeinae) de bosque seco de Colombia. Biota Colombiana, 16(1), 36-44.

Hedrick, B. P., Heberling, J. M., Meineke, E. K., Turner, K. G., Grassa, C. J., Park, D. S., Kennedy, J., Clarke, J. A., Cook, J. A., Blackburn, D. C. 2020. Digitization and the future of natural history collections. BioScience, 7o(3), 243-251. Disponible en: https://doi.org/10.7287/peerj.preprints.27859v1

Hernández-Camacho, J., Hurtado-Guerra, A., Ortiz-Quijano, R., Walschburger, T. 1992. Unidades biogeográficas de Colombia. La diversidad biológica de Iberoamérica, 1: 105-151.

IAVH. 2020. Registro Único Nacional de Colecciones Biológicas. Listado de colecciones registradas. Disponible en: http://rnc. humboldt.org.co/wp/,http://rnc.humboldt.org.co/admin/ index.php/registros/detail/1396.

IDEAM, IGAC, IAvH, Invemar, Sinchi, IIAP. 2007. Ecosistemas continentales, costeros y marinos de Colombia IDEAM, IGAC, IAvH, IIAP, Invemar, y Sinchi. Bogotá, D.C.

IDEAM, IGAC, IAvH, Invemar, Sinchi, IIAP. 2017. Mapa de Ecosistemas Continentales, Costeros y Marinos Escala 1: 100.ooo. Bogotá. DC, Colombia.

Jaramillo-Rivera, D. 2020. Filogeografía de Eurysternus foedus Guérin-Méneville, 1830 (Coleoptera: Scarabaeidae), en tres provincias biogeográficas de Colombia. Trabajo de Grado en Biología, Universidad de Caldas, Facultad de Ciencias Exactas y Naturales, Programa de Biología, Manizales - Caldas.

Lopez-Collado, J., Cruz-Rosales, M., Vilaboa-Arroniz, J., Martínez-Morales, I., Gonzalez-Hernandez, H. 2017. Contribution of dung beetles to cattle productivity in the tropics: A stochasticdynamic modeling approach. Agricultural Systems, 155, 78-87. Disponible en: https://doi.org/10.1016/j.agsy.2017.05.001 
Martínez-Revelo, D. E., Torres, E., Neita-Moreno, J. C. 2020. The genus Cryptocanthon (Coleoptera: Scarabaeidae) in Colombia: description of new species, geographical distribution and conservation. Revista mexicana de biodiversidad, 91, e913156. Disponible en: https://doi. org/10.22201/ib.20078706e.2020.91.3156.

Montaño, M., Meza, A. M., Dias, L. 2012. La colección entomológica CEBUC y su potencial como colección de referencia de insectos acuáticos. Boletín Científico Museo de Historia Natural, 16(2), 173-184.

Mora-Ambriz, L., Fuentes-Moreno, H. 2006. El Laboratorio de Colecciones Biológicas de la Universidad del Mar: perspectivas de una colección regional. Ciencia y Mar, 28, 34-37.

Morales-Castaño, I. T., Medina, C. A. 2009. Insectos de la Orinoquía colombiana: evaluación a partir de la Colección Entomológica del Instituto Alexander von Humboldt (IAvH). Biota Colombiana, 10(1 y 2).

Navarro-Quintero, I., Salazar-Salinas, L. F. 2019. Afinidades bióticas entre las provincias biogeográficas Chocó- Darién y Magdalena utilizando como modelo los escarabajos coprófagos (Coleoptera: Scarabaeinae). Trabajo de Grado en Biología, Universidad de Caldas, Facultad de Ciencias Exactas y Naturales, Programa de Biología, Manizales - Caldas.

Nichols, E., Spector, S., Louzada, J., Larsen, T., Amezquita, S., Favila, M., Network, T. S. R. 2008. Ecological functions and ecosystem services provided by Scarabaeinae dung beetles. Biological conservation, 141(6), 1461-1474. Disponible en: https://doi.org/10.1016/j.biocon.2008.04.011

Noriega, J. A., Camero, E., Arias-Buriticá,J., Pardo-Locarno, L. C., Montes, J. M., Acevedo, A. A., Esparza, A., Ordóñez, B. M., Garcia, H., Solís, C. 2015. Grado de cobertura del muestreo de escarabajos coprófagos (Coleoptera: Scarabaeidae: Scarabaeinae) en Colombia. Revista de Biología Tropical, 63(1), 97-125. Disponible en: https://doi.org/10.15517/rbt.v63i1.13323 
Nunes, R. V., Vaz-de-Mello, F. Z. 2019. Taxonomic revision of Dichotomius (Cephagonus) Luederwaldt 1929 and the taxonomic status of remaining Dichotomius Hope 1838 subgenera (Coleoptera: Scarabaeidae: Scarabaeinae: Dichotomiini). Journal of Natural History, 53(37-38), 2231-2351. Disponible en: https://doi.org/10.1080/00222933.2019.1692088

Pardo-Diaz, C., Toro, A. L., Tovar, S. A. P., Sarmiento-Garcés, R., Herrera, M. S., Salazar, C. 2019. Taxonomic reassessment of the genus Dichotomius (Coleoptera: Scarabaeinae) through integrative taxonomy. PeerJ, 7, e7332. Disponible en: https://doi. org/10.7717/peerj.7332

Pyke, G. H., Ehrlich, P. R. 2010. Biological collections and ecological/environmental research: a review, some observations and a look to the future. Biological reviews, 85(2), 247-266. Disponible en: https://doi.org/10.1111/j.1469185X.2009.00098.X

Rodríguez, N., Armenteras, D., Morales, M., Romero, M. 2004. Ecosistemas de los Andes colombianos: Instituto de Investigación de Recursos Biológicos Alexander von Humboldt.

Rossini, M., Vaz-de-Mello, F. Z., Zunino, M. 2018. A taxonomic revision of the New World Onthophagus Latreille, 1802 (Coleoptera: Scarabaeidae: Scarabaeinae) of the osculatii species-complex, with description of two new species from South America. Journal of Natural History, 52(9-10), 541-586. Disponible en: https://doi.org/10.1080/00222933.2018.1437230

SIB-Colombia. (2021). Sistema de la Información sobre Biodiversidad de Colombia. Disponible en: https://sibcolombia. net/estandares/

Slade, E. M., Riutta, T., Roslin, T., Tuomisto, H. L. 2016. The role of dung beetles in reducing greenhouse gas emissions from cattle farming. Scientific Reports, 6(1), 1-9. Disponible en: https://doi.org/10.1038/srep18140 
Suarez, A. V., Tsutsui, N. D. 2004. The value of museum collections for research and society. BioScience, 54(1), 66-74.

Tarasov, S., Dimitrov, D. 2016. Multigene phylogenetic analysis redefines dung beetles relationships and classification (Coleoptera: Scarabaeidae: Scarabaeinae). BMC evolutionary biology, 16(1), 257. Disponible en: https:// doi.org/10.1186/s12862-016-0822-X

Team, Q. D. 2018. QGIS Geographic Information System (Version QGIS 3.4.12 'Madeira'). Retrieved from https://www.qgis.org

Vallejo, M. Y., Acosta, A. 2005. Aplicación de indicadores de conocimiento sobre biodiversidad para el diagnóstico y comparación de colecciones biológicas. Nova, 3(4), 48-57. Disponible en: https://doi.org/10.22490/24629448.336

Vaz-de-Mello, F. Z., Edmonds, W., Ocampo, F. C., Schoolmeesters, P. 2011. A multilingual key to the genera and subgenera of the subfamily Scarabaeinae of the New World (Coleoptera: Scarabaeidae). Zootaxa, 2854(1), 1-73.

Vélez, E. D., Calderón-Espinosa, M. L., Ramírez-Pinilla, M. P., Castaño, M., Reyes, Á. M., Albarracín, R., Liévano, A., Raz, L. 2012. Difusión de datos biológicos en la red como apoyo a la educación ambiental, investigación científica y conservación de la biodiversidad en Colombia. e-colabora "Revista de ciencia, educación, innovación y cultura apoyadas por redes de tecnología avanzada”, 2(4), 49-57.

Vieira, M. K., Vaz-de-Mello, F. Z., Silva, F. A. 2020. A taxonomic revision of the Canthon subgenus Pseudepilissus Martínez, 1954 (Coleoptera: Scarabaeidae: Scarabaeinae). Insect Systematics \& Evolution, 51(4), 696-752. Disponible en: https://doi.org/10.1163/1876312X-00001023

Winker, K. 2004. Natural history museums in a postbiodiversity era. BioScience, 54(5), 455-459. 
Zhang, S.-Q., Che, L.-H., Li, Y., Liang, D., Pang, H., Ślipiński, A., Zhang, P. 2018. Evolutionary history of Coleoptera revealed by extensive sampling of genes and species. Nature communications, 9(1), 1-11. Disponible en: https://doi. org $/ 10.1038 / \mathrm{s} 41467-017-02644-4$ 


\section{Anexos}

Anexo 1: Fotografías de algunos de los géneros de escarabajos copronecrófagos depositados en la Colección Entomológica del Programa de Biología de la Universidad de Caldas (CEBUC). Fotografías facilitadas y modificadas a partir del libro de Cultid-Medina et al. (2012). Foto de Digitonthophagus tomada por Sebastián Villada-Bedoya, 2019 (C).

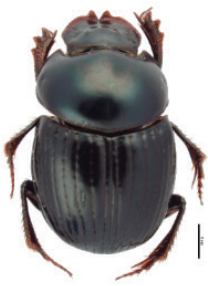

Canthidium
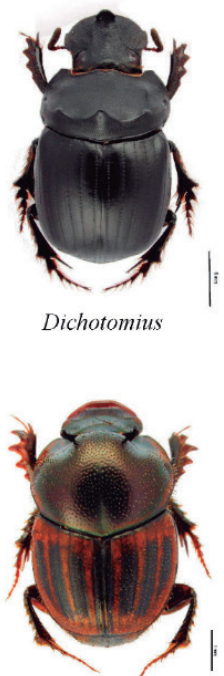

Onthophagus

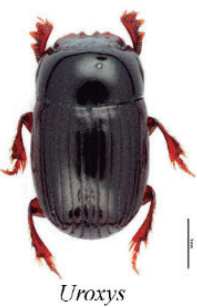

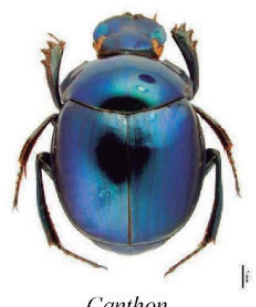

Canthon

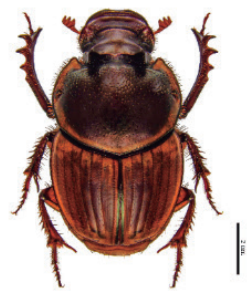

Digitonthophagus

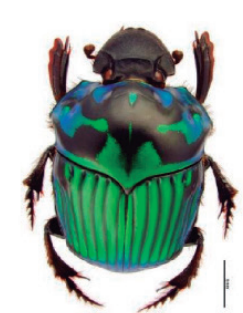

Oxysternon
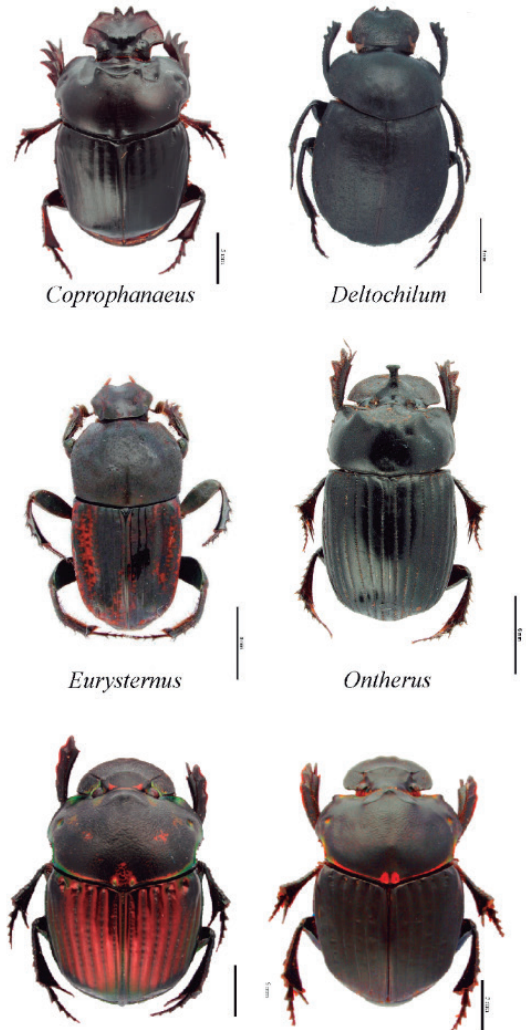

Phanaeus

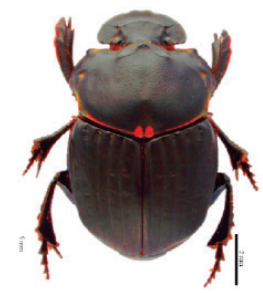

Sulcophanaeus 